\title{
Saliva pools for screening of human cytomegalovirus using real-time PCR
}

\author{
Cláudia Fernandes ${ }^{1}$ - Augusta Marques ${ }^{1} \cdot$ Maria de Jesus Chasqueira ${ }^{1} \cdot$ Mónica Cró Braz ${ }^{2} \cdot$ Ana Rute Ferreira $^{2}$. \\ Ana Serrão Neto ${ }^{2}$. Cândida Mendes ${ }^{3} \cdot$ David Lito $^{3} \cdot$ Maria-Favila Menezes $^{4} \cdot$ Maria José Sousa $^{4} \cdot$ Paulo Paixão $^{1}$
}

Received: 12 June 2020 / Revised: 9 September 2020 / Accepted: 9 October 2020 / Published online: 14 October 2020

(C) The Author(s) 2020

\begin{abstract}
Human cytomegalovirus (HCMV) is the leading congenital infection agent in the world. The importance of screening this infection has been debated, as $10-15 \%$ of the asymptomatic newborns with HCMV at birth will present late sequelae. The aim of this study was to test the feasibility of using saliva pools from newborns in a screening program for congenital HCMV infection, in two Portuguese hospitals. The screening was based on the use of pools of 10 saliva samples for detection of viral DNA by real-time PCR. Whenever there was a positive pool, the samples were tested individually, and for each positive sample the result was confirmed with a urine sample collected in the first 2 weeks of life. The study involved 1492 newborns. One hundred and fifty pools were screened, with 14 positive results in saliva, but only 10 were confirmed in urine samples, giving a prevalence of congenital HCMV infection in both hospitals of $0.67 \%$ (CI95\% 0.36 to $1.23 \%$ ).

Conclusion: The overall prevalence of congenital HCMV infection in both hospitals was $0.67 \%$. The use of saliva pools proved to be effective for the screening of this congenital infection, allowing timely screening and confirmation in a large population, with associated cost reduction.
\end{abstract}

What is Known:

- Newborn screening for HCMV is desirable.

- Saliva is a good and practical sample.

What is New:

- The feasibility of using saliva pools for a large-scale screening.

- The cost reduction of this strategy.

Keywords HCMV $\cdot$ Newborn $\cdot$ Cost reduction $\cdot$ Pools

Communicated by Nicole Ritz

Electronic supplementary material The online version of this article (https://doi.org/10.1007/s00431-020-03842-x) contains supplementary material, which is available to authorized users.

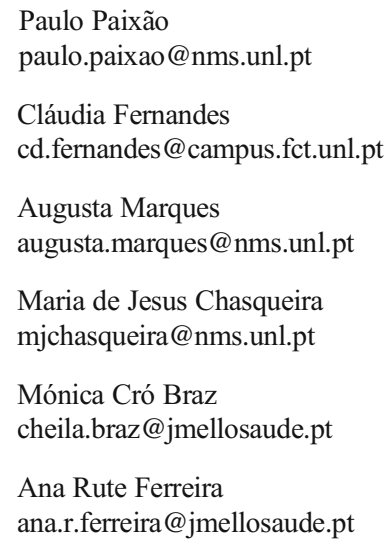

\author{
Ana Serrão Neto \\ ana.neto@jmellosaude.pt \\ Cândida Mendes \\ Mendesscandida@sapo.pt \\ David Lito \\ dmlito@gmail.com \\ Maria-Favila Menezes \\ mmnezes@germanodesousa.com \\ Maria José Sousa \\ mariajsousa@cm-lab.com
}

Extended author information available on the last page of the article 


\section{Abbreviations}

HCMV Human cytomegalovirus

SNHL Sensorineural hearing loss

\section{Introduction}

Human cytomegalovirus (HCMV) is the leading congenital infection agent, with an incidence ranging between 0.2 and $2 \%$ worldwide $[1,2]$. In industrialized countries, an estimated 0.6 to $0.7 \%$ of newborns are congenitally infected with this virus [1, 3-5]. Long-term sequelae of this infection affect more children than others with better-known clinical conditions, such as Down syndrome, foetal alcohol syndrome, or spina Bifida. Ongoing special care these children require for life results in substantial economic burden [6]. Strategies to reduce this burden have been debated in the last two decades, including earlier identification of infection through maternal or newborn screening $[5,7]$.

Maternal screening during pregnancy is not currently advised, and some concerns are raised. Indeed, not only counselling programs and treatment have not yet been proven effective as some adverse effects can be associated with this screening [8].

Between 85 and $90 \%$ of newborns with laboratoryconfirmed congenital infection will be asymptomatic at birth or present nonspecific symptoms. However, 10 to $15 \%$ of these asymptomatic newborns may develop late sequelae at up to 5 years of age such as neurosensorial hearing loss (SNHL), decreased visual acuity, and progressive neurological changes $[9,10]$. As neonatal screening for this infection is not currently implemented, a large number of cases of congenital HCMV infection will not be detected. The only way to detect all cases of congenital infection with this virus would be to routinely screen all newborns in the first 2 weeks of life. The implementation of this procedure would enable the monitoring of children with asymptomatic infection at birth, with proven benefit of an early intervention $[11,12]$. However, one of the constraints of implementing routine neonatal screening is the associated cost to perform a diagnostic test for each newborn.

Currently, when a congenital infection is suspected, the diagnosis is performed using urine or saliva samples collected within the first 3 weeks of the newborn's life. In order to circumvent the elevated cost of the screening, our team previously developed a urine pool methodology for congenital HCMV: based on the fact that the viral shed in the urine is high, it can be detected by real-time PCR even if the positive sample is diluted $1 / 20$. This method, which was shown to have similar sensitivity and specificity to cell culture (reference method), allows a significant reduction in reagent cost and execution time. This approach may be feasible for universal screening of this infection [13]. However, urine collection through a paediatric collection bag is a time-consuming procedure with some additional disadvantages, like skin irritation due the glue used to hold the bag, perineum irritation, or delayed collection due to inadequate diuresis or child stress, $[14,15]$. Therefore, this collection method may cause discomfort to the child and consequently to parents, preventing its use in a universal screening program.

The possibility of implementing a screening program using saliva samples has been studied in recent years, as saliva is much simpler to collect [16-19]. A recent study in our laboratory has shown that the real-time PCR technique using saliva pools has high sensitivity and specificity (100\%) when compared with single sample testing and could easily be applied to large-scale studies, enabling the identification of most newborns with congenital HCMV infection at a significantly reduced cost [20].

The aim of this study was to test the feasibility of using saliva samples from newborns and a pool methodology for a screening program. Secondly, we intended to estimate the prevalence of HCMV congenital infection in two Portuguese hospitals.

\section{Materials and methods}

Population The study population included 1492 newborns aged between 1 day and 2 weeks, of which 748 and 744 were born respectively at Hospital CUF Descobertas and at Hospital Vila Franca de Xira (Portugal). The screening was conducted for 8 months, and samples were collected between October 2018 and May 2019.

Samples Saliva samples were collected using breakable rod rayon swabs (FLmedical, Torreglia, Italy) and were always carried out before breastfeeding or at least $1 \mathrm{~h}$ after the last meal. The swab was packaged in a $3-\mathrm{mL}$ tube with $500-\mu \mathrm{L}$ RPMI medium (Life Technologies, Paisley, UK). After collection the tubes were stored at $4{ }^{\circ} \mathrm{C}$ until processing. Urine samples were requested to confirm congenital infection if a positive result was obtained with a saliva sample. These were collected using paediatric urine collection bags, then placed in 50-mL flasks and stored at $4{ }^{\circ} \mathrm{C}$ until processing.

All samples were sent to the Unit of Infection of the Faculdade de Ciências Médicas|Nova Medical School (FCM|NMS) for processing.

Sample processing The samples were centrifuged at $948 \times g$ (Hettich, Routine $380 \mathrm{R}$ ) for $5 \mathrm{~min}$ and transferred in a biosafety chamber into properly identified freezing tubes. Disinfection procedures were performed between each sample transfer and every five samples; the work area and material were submitted to UV radiation to avoid contamination. Pools were prepared mixing $20 \mu \mathrm{L}$ of each 10 saliva samples in a 1.5-mL eppendorf tube. Genomic DNA was extracted using 
the Purelink Genomic DNA commercial kit (Invitrogen, Carlsbad, USA), according to the manufacturer's instructions and stored at $-20{ }^{\circ} \mathrm{C}$ until use. HCMV DNA was amplified and detected by qPCR on the Applied Biosystems 7500 Fast Real-Time PCR System (Applied Biosystems, Foster City, USA) using an "in-house" method, detailed elsewhere, with $100 \%$ sensitivity and specificity when compared with individual samples $[13,20]$. Each pool was tested according to the algorithm described in Fig. 1.

Positive and negative controls were added in each series. An internal control was made for each pool and sample tested by adding $2 \mu \mathrm{L}$ of AD169 strain DNA ( 69,000 copies $/ \mathrm{mL})$ to test for the presence of inhibitory substances. All pools and samples were tested in duplicated.

Quantitative analysis of pools and individual positive samples of saliva and urine were performed using a calibration curve made with four 1:10 serial dilutions of the 1st World Health Organization International Standard, NIBSC 09/162, for HCMV nucleic acid amplification techniques with an initial concentration of $5 \times 10^{6}$ International Units per millilitre $(\mathrm{IU} / \mathrm{mL})$. Quantifications were obtained by extrapolating the $\mathrm{CT}$ results into the calibration curve, and the results were expressed in IU $/ \mathrm{mL}$.

\section{Results}

In this study we grouped 1492 saliva samples into 150 pools of 10 samples each. Of these, 140 tested negatives. Ten positive pools were obtained and, after individual testing of each sample that composed them (following the algorithm described in Fig. 1), we identified 14 positive saliva samples (Table 1). Four pools had two positive samples simultaneously.

In order to verify the agreement between the methodology used to perform this screening and the reference method (individual testing), samples from 10 pools with negative result were tested individually and obtained concordant results in all samples analysed (100\% agreement with the reference test).
Table 1 Real-time PCR qualitative results of saliva pools and positive individual samples

\begin{tabular}{lllll}
\hline 10-pool & & & \multicolumn{2}{l}{ Positive saliva samples } \\
\cline { 5 - 5 } No. & $\mathrm{UI} / \mathrm{mL}$ & & No. & $\mathrm{UI} / \mathrm{mL}$ \\
\hline POOL 7 & $1.7 \times 10^{3}$ & & CMV12A & $7.4 \times 10^{3}$ \\
& & & CMV21A & $7.4 \times 10^{1}$ \\
POOL 13 & $1.8 \times 10^{6}$ & & CMV82 & $1.5 \times 10^{6}$ \\
POOL 15 & $1.2 \times 10^{7}$ & & CMV57A & $8.2 \times 10^{7}$ \\
& & & CMV58A & $1.3 \times 10^{3}$ \\
POOL 21 & $5.2 \times 10^{3}$ & & CMV103A & $4.2 \times 10^{4}$ \\
POOL 51 & $6.0 \times 10^{5}$ & & CMV253 & $8.4 \times 10^{5}$ \\
POOL 57 & $1.3 \times 10^{5}$ & & CMV305A & $6.1 \times 10^{4}$ \\
& & & CMV307A & $2.0 \times 10^{6}$ \\
POOL 78 & $2.5 \times 10^{3}$ & & CMV420A & $1.8 \times 10^{1}$ \\
& & & CMV422A & $8.8 \times 10^{4}$ \\
POOL 90 & $7.7 \times 10^{2}$ & & CMV481A & $6.9 \times 10^{3}$ \\
POOL 106 & $4.4 \times 10^{4}$ & & CMV566A & $6.9 \times 10^{5}$ \\
POOL 131 & $6.8 \times 10^{3}$ & & CMV633 & $3.1 \times 10^{4}$ \\
\hline
\end{tabular}

For the confirmatory diagnosis, urine samples were requested from the newborns who tested positive with the saliva sample. Of the 14 positive saliva samples, 10 had congenital infection confirmed by the urine analysis and the remaining four were false positive results. These false positive saliva samples had significantly lower HCMV viral loads than the other samples associated with congenital infection (Table 2). Therefore, of the 1492 newborns screened, 10 had confirmed congenital infection, which represents a prevalence of $0.67 \%$ in the two hospitals under study (95\% confidence interval, exact binomial method: $0.32-1.23 \%$ ).

\section{Discussion}

In a nationwide study performed in 2004, the prevalence of HCMV congenital infection in Portugal was described as

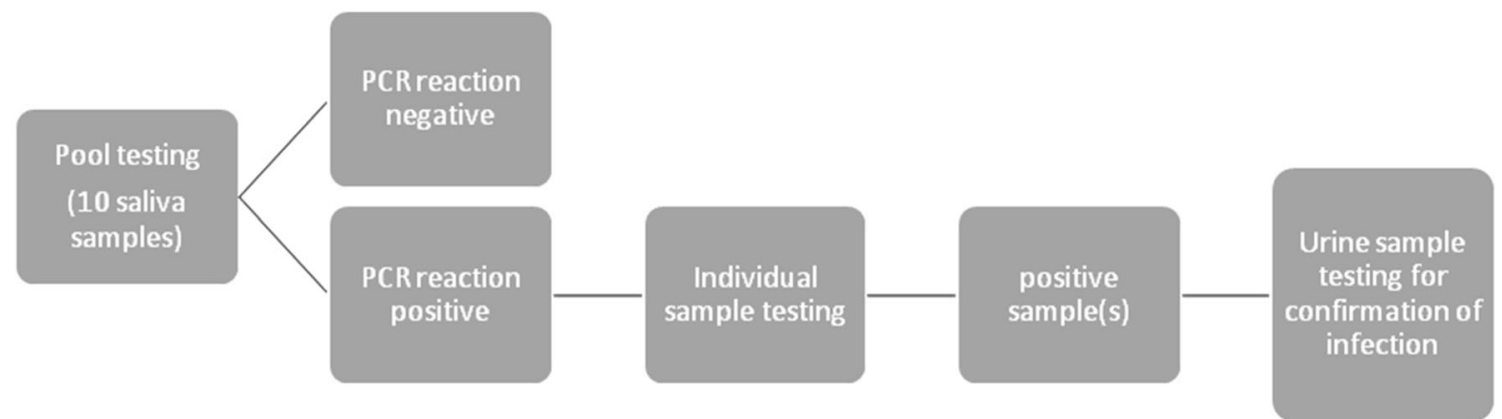

Fig. 1 Algorithm used for detection and identification of HCMV DNA positive specimens in 10--pool saliva samples. When positive, each sample of the pool was tested individually. Upon identification of the positive sample(s) in order to confirm congenital HCMV infection, a urine sample was requested and tested by the same technique as the saliva sample 


\begin{tabular}{lll} 
Table 2 Quantification & & \\
\cline { 2 - 3 } $\begin{array}{l}\text { values in IU/mL for } \\
\text { individual positive saliva } \\
\text { samples and }\end{array}$ corresponding 20 sample & Nositive saliva samples & 20-pool \\
pools (NT - not tested; & CMV57A \\
\cline { 2 - 3 } Neg - negative) & CMV82 & $1.2 \times 10^{7}$ \\
& CMV566A & $2.3 \times 10^{6}$ \\
& CMV253 & $5.3 \times 10^{5}$ \\
& CMV307A & $1.7 \times 10^{5}$ \\
& CMV422A & $8.1 \times 10^{4}$ \\
& CMV305A & $1.3 \times 10^{4}$ \\
& CMV633 & $7.8 \times 10^{3}$ \\
& CMV103A & $2.6 \times 10^{3}$ \\
& CMV481A & $2.5 \times 10^{3}$ \\
& CMV12A & $4.7 \times 10^{2}$ \\
& CMV58A & $4.4 \times 10^{2}$ \\
& CMV21A & $\mathrm{NT}$ \\
& CMV420A & $\mathrm{Neg}$ \\
& & $\mathrm{Neg}$ \\
\hline
\end{tabular}

$1.05 \%$, higher than the prevalence in other European countries [13]. In the current study we found a prevalence of $0.67 \%$, a value similar to the birth prevalence of congenital CMV infection described for developed countries [1,3-5]. Although the present study was limited to two hospitals in Lisbon and had a low number of samples (giving a 95\% confidence interval of $0.32-1.23 \%$ ), it is possible that the prevalence has been declining in the past years. In 2011, a Portuguese study by Lopo et al. found a lower seroprevalence for HCMV in the central coastal zone, corresponding with our study population; this can explain our results. [21].

Pool methodology for urine samples was proven accurate in a previous study [13]. However, collecting a saliva sample is simpler, faster, and less uncomfortable for the newborns, and therefore better suited for a universal screening program. Concerns about false positive results with these samples have been raised because virus transmission during breastfeeding may occur. Several articles reported the excretion of this virus in the breast milk of healthy women [22, 23]. To prevent these false-positive during screening programs an approach would be to instruct health professionals to collect the sample before the first breastfeeding, although this is not always possible. Therefore, the confirmation of a positive saliva sample should always be performed by viral detection in a urine sample within the first 2 weeks of life [24], which was done with all the cases in our study. This is, however, the general rule for all the saliva samples, regardless if they are tested individually or if first screened by a pool method. In this study, four false positive results were detected with our saliva pool methodology (i.e., the urine samples asked for confirmation were negative in these four children). These false-positive samples had significantly lower HCMV viral loads than the other samples associated with congenital infection, which is in agreement with another study [22], suggesting a dilution effect of the breast milk on the newborn's saliva and eventually a lower viral load on breast milk. Therefore, the low viral load of these samples reinforces the idea that these saliva-positive/urine-negative samples were in fact false-positive saliva results and not false-negative urine results. Nevertheless, these false-positive results were not related with the pool approach but rather, as explained before, by using saliva instead of urine samples.

Although the reduction in reagent cost and execution time by the pool method was not quantified in the Results section, the advantage of this strategy is obvious: ten samples are screened with a single PCR, instead of testing ten individual samples. With a prevalence around $0.7 \%$, that means that only $1-2$ pools will be positive in 20 tested pools, which means that only $30-40$ PCR tests will be need to screen 200 newborns, thus reducing the associated cost of this screening when compared with 200 individual tests by the conventional approach. The reason for choosing pools of 10 instead of 20 samples in the current study was a practical decision, to have a faster answer with the pool strategy (results should be ready within 1 week, allowing the confirmatory urine sample to be collected within the first 2 weeks of life). In fact, the 10-pool approach can be used either in hospitals with a few hundred deliveries a year or in hospitals with thousands of newborns. For example, in a hospital with 500-600 births/year, we would have to wait 2 weeks to collect 20 samples for the 20pool strategy, which would prevent us from giving an answer within the 1-week period indicated above and to get the urine sample for confirmation within the first 2 weeks.

Screening for HCMV infection in pregnancy is not advised for various reasons, such as lack of effective treatment programs and the difficult prognosis regarding the congenital infection sequelae [8]. Thus, only through a universal screening program for congenital HCMV infection in newborns would it be possible to diagnose all cases of infection, especially in the asymptomatic ones. Routine implementation of this procedure would enable early monitoring of children with asymptomatic birth infection, particularly regarding the risk of developing late sequelae such as SNHL and progressive neurological changes. It has been proven that this early intervention benefits quality of life of these children [5, 11, 25].

\section{Conclusions}

Using this saliva pool methodology, we were able to screen 1492 newborns for an 8-month period, with a significant reduction in reagent cost and execution time. These results, combined with the lack of an effective vaccine, open the possibility of using this approach on a large-scale screening for HCMV congenital infection, allowing an early intervention 
which can benefit the affected children's life quality. Nevertheless, the feasibility of this approach needs to be confirmed by a larger multicenter study, which is currently underway.

Authors' contributions CF: material preparation, data analysis, first and second draft of the manuscript, and approval of the final manuscript; AM: material preparation, technical supervision and approval of the final manuscript; MJC: supervision in the analysis of the results, critical revision of the manuscript, and approval of the final manuscript; $\mathrm{MB}, \mathrm{AF}$, and $\mathrm{CM}$ : data collection and approval of the final manuscript; AN and DL: critical revision of the manuscript and approval of the final manuscript; MFM and MJS: organization of the sample collection, critical revision of the manuscript, and approval of the final manuscript. PP: designed the study concept, review and editing of the manuscript, and approval of the final manuscript.

Data availability The datasets used and/or analysed during the current study are available from the corresponding author on request.

\section{Compliance with ethical statements}

Conflict of interest The authors declare that they have no conflict of interest.

Ethical approval The study was approved by the ethics committees of the FCM|NMS and the two participant hospitals.

Informed consent Written informed consent was obtained from a parent for their newborn's enrolment in the study.

Open Access This article is licensed under a Creative Commons Attribution 4.0 International License, which permits use, sharing, adaptation, distribution and reproduction in any medium or format, as long as you give appropriate credit to the original author(s) and the source, provide a link to the Creative Commons licence, and indicate if changes were made. The images or other third party material in this article are included in the article's Creative Commons licence, unless indicated otherwise in a credit line to the material. If material is not included in the article's Creative Commons licence and your intended use is not permitted by statutory regulation or exceeds the permitted use, you will need to obtain permission directly from the copyright holder. To view a copy of this licence, visit http://creativecommons.org/licenses/by/4.0/

\section{References}

1. Dollard SC, Grosse SD, Ross DS (2007) New estimates of the prevalence of neurological and sensory sequelae and mortality associated with congenital cytomegalovirus infection. Rev Med Virol 17(5):355-363

2. Lanzieri TM, Dollard SC, Bialek SR, Grosse SD (2014) Systematic review of the birth prevalence of congenital cytomegalovirus infection in developing countries. Int J Infect Dis 22:44-48

3. Kenneson A, Cannon MJ (2007) Review and meta-analysis of the epidemiology of congenital cytomegalovirus (CMV) infection. Rev Med Virol 17(4):253-276
4. Ludwig A, Hengel H (2009) Epidemiological impact and disease burden of congenital cytomegalovirus infection in Europe. Eurosurveillance 14(9):19140

5. Cannon MJ, Griffiths PD, Aston V, Rawlinson WD (2014) Universal newborn screening for congenital CMV infection: what is the evidence of potential benefit? Rev Med Virol 24(5):291-307

6. Cannon MJ, Davis KF (2005) Washing our hands of the congenital cytomegalovirus disease epidemic. BMC Public Health 5(1):70

7. Grosse SD, Dollard S, Ross DS, Cannon M (2009) Newborn screening for congenital cytomegalovirus: options for hospitalbased and public health programs. J Clin Virol 46:S32-S36

8. Vauloup-Fellous C, Picone O, Cordier AG, Parent-du-Châtelet I, Senat MV, Frydman R, Grangeot-Keros L (2009) Does hygiene counseling have an impact on the rate of CMV primary infection during pregnancy? Results of a 3-year prospective study in a French hospital. J Clin Virol 46:S49-S53

9. Mocarski ES Jr, Shenk T, Pass RF (2013) Cytomegaloviruses. In: Knipe DM, Howley PM (eds) Fields virology. Lippincott Williams \& Wilkins, Philadelphia, pp 1960-2014

10. van Zuylen WJ, Hamilton ST, Naing Z, Hall B, Shand A, Rawlinson WD (2014) Congenital cytomegalovirus infection: clinical presentation, epidemiology, diagnosis and prevention. Obstet Med 7(4):140-146

11. Barbi M, Binda S, Caroppo S (2006) Diagnosis of congenital CMV infection via dried blood spots. Rev Med Virol 16(6):385-392

12. Paixão P, Almeida S, Videira PA, Ligeiro D, Marques T (2012) Screening of congenital cytomegalovirus infection by real-time PCR in urine pools. Eur J Pediatr 171(1):125-129

13. Paixão $\mathrm{P}$, Almeida $\mathrm{S}$, Gouveia $\mathrm{P}$, Binda $\mathrm{S}$, Caroppo $\mathrm{S}$, Barbi $\mathrm{M}$ (2005) Diagnosis of congenital cytomegalovirus infection by detection of viral DNA in urine pools. J Virol Methods 128(1-2):1-5

14. Waters A, Jennings K, Fitzpatrick E, Coughlan S, Molloy EJ, De Gascun CF, Knowles SJ (2014) Incidence of congenital cytomegalovirus infection in Ireland: implications for screening and diagnosis. J Clin Virol 59(3):156-160

15. Ross SA, Ahmed A, Palmer AL, Michaels MG, Sánchez PJ, Stewart A, Boppana SB (2015) Urine collection method for the diagnosis of congenital cytomegalovirus infection. Pediatr Infect Dis J 34(8):903-905

16. Yamamoto AY, Mussi-Pinhata MM, Marin LJ, Brito RM, Oliveira PFC, Coelho TB (2006) Is saliva as reliable as urine for detection of cytomegalovirus DNA for neonatal screening of congenital CMV infection? J Clin Virol 36(3):228-230

17. Boppana SB, Ross SA, Shimamura M, Palmer AL, Ahmed A, Michaels MG et al (2011) Saliva polymerase-chain-reaction assay for cytomegalovirus screening in newborns. N Engl J Med 364(22): 2111-2118

18. Barkai G, Roth DAE, Barzilai A, Tepperberg-Oikawa M, Mendelson E, Hildesheimer M, Kuint J (2014) Universal neonatal cytomegalovirus screening using saliva-report of clinical experience. J Clin Virol 60(4):361-366

19. Ross SA, Ahmed A, Palmer AL, Michaels MG, Sánchez PJ, Bernstein DI, Boppana SB (2014) Detection of congenital cytomegalovirus infection by real-time polymerase chain reaction analysis of saliva or urine specimens. J Infect Dis 210(9):1415-1418

20. Silva J, Fernandes C, Marques A, Maria AT, Correia C, Tuna ML, Chasqueira MJ, Paixão P (2020) Evaluation of saliva pools method for detection of congenital human cytomegalovirus infection. J Virol Methods 275:113759

21. Lopo S, Vinagre E, Palminha P, Paixão MT, Nogueira P, Freitas MG (2011) Seroprevalence to cytomegalovirus in the Portuguese population, 2002-2003. Euro Surveill 16(25) 
22. Ross SA, Michaels MG, Ahmed A, Palmer AL, Palmer AL, Bernstein DI, Feja F, Stewart A, Boppana SB, Fowler KB (2018) Contribution of breastfeeding to false-positive saliva polymerase chain reaction for newborn congenital cytomegalovirus screening. J Infect Dis 217(10):1612-1615

23. Azenkot T, Zaniello B, Green ML, Selke S, Huang ML, Magaret A, Johnston C (2019) Cytomegalovirus shedding from breastmilk and mucosal sites in healthy postpartum women: a pilot study. J Med Virol 91(5):894-898

24. Luck SE, Wieringa JW, Blázquez-Gamero D, Henneke P, Schuster K, Butler K, Heath P (2017) Congenital cytomegalovirus: a
European expert consensus statement on diagnosis and management. Pediatr Infect Dis J 36(12):1205-1213

25. Kimberlin DW, Lin CY, Sánchez PJ, Demmler GJ, Dankner W, Shelton M, Soong SJ (2003) Effect of ganciclovir therapy on hearing in symptomatic congenital cytomegalovirus disease involving the central nervous system: a randomized, controlled trial. J Pediatr 143(1):16-25

\section{Affiliations}

\section{Cláudia Fernandes $^{1}$ - Augusta Marques ${ }^{1} \cdot$ Maria de Jesus Chasqueira ${ }^{1} \cdot$ Mónica Cró Braz ${ }^{2}$ - Ana Rute Ferreira ${ }^{2}$.} Ana Serrão $\mathrm{Neto}^{2} \cdot$ Cândida Mendes $^{3} \cdot$ David Lito $^{3} \cdot$ Maria-Favila Menezes $^{4} \cdot$ Maria José Sousa $^{4} \cdot$ Paulo Paixão $^{1}$

1 Centro de Estudos de Doenças Crónicas, CEDOC, Faculdade de Ciências Médicas|NOVA Medical School, Campo Mártires da Pátria, 130, 1169-056 Lisbon, Portugal

2 Serviço de Pediatria, Hospital CUF Descobertas, Rua Mário Botas, 1998-018 Lisbon, Portugal
3 Serviço de Pediatria, Hospital Vila Franca de Xira, Estrada Carlos Lima Costa no. 2, 2600-009 Vila Franca de Xira, Portugal

4 Centro de Medicina Laboratorial Germano de Sousa, Rua Cupertino de Miranda, 1600-513 Lisbon, Portugal 